\title{
REDD: DESAFIO E OPORTUNIDADE DE IMPLEMENTAÇÃO DE POLÍTICA PÚBLICA DE VALORIZAÇÃO DO CERRADO NO ESTADO DE GOIÁS
}

\author{
REDD: CHALLENGE AND OPPORTUNITY TO IMPLEMENT PUBLIC POLICY \\ FOR VALUATION OF THE CERRADO IN GOIÁS STATE
}

\author{
REDD: DESAFÍO Y OPORTUNIDAD PARA IMPLEMENTAR POLÍTICAS \\ PÚBLICAS PARA LA VALORACIÓN DEL CERRADO EN EL ESTADO DE GOIÁS
}

\author{
Juliana Ferreira Leite - Universidade Federal de Goiás - Goiânia - Goiás - Brasil \\ julianafleite.geo@gmail.com \\ Laerte Guimarães Ferreira - Universidade Federal de Goiás - Goiânia - Goiás - Brasil \\ laerte@iesa.ufg.br
}

\section{Resumo}

Este texto delineia as perspectivas e os desafios para uma aplicação de Pagamento por Serviços Ambientais (PSA) no estado de Goiás, tendo como foco específico a implementação de um mecanismo que está em grande debate no momento no âmbito da Convenção do Clima: a Redução de Emissões por Desmatamento e Degradação Florestal (Reducing Emissions from Deforestation and Forest Degradation - REDD, em inglês). Numa abordagem qualitativa, utilizam-se fontes bibliográficas (secundárias) para a discussão do objeto abordado, e conclui-se que, apesar dos desafios e das dificuldades técnicas e operacionais que ainda precisam ser enfrentados e superados, o REDD pode ser viável e eficaz como um mecanismo de redução compensada do desmatamento e de conservação do Cerrado no Estado de Goiás.

Palavras-chave: mudanças climáticas REDD, bioma Cerrado, estado de Goiás.

\section{Abstract}

This paper outlines the prospects and challenges concerning the Payment for Environmental Services (PSA) in Goias State - Brazil. Specifically, it focuses on the implementation of a mechanism that is currently in great debate under the Climate Convention: Reducing Emissions from Deforestation and Forest Degradation (REDD). This issue is addressed through a qualitative approach, considering a variety of secondary literature sources. We conclude that, in spite of the challenges and technical and operational difficulties to be faced and overcome, REDD may be feasible and effective as a mechanism of compensated reduction of deforestation and conservation of the Cerrado in Goias State.

Keywords: climate change, REDD, Cerrado biome, Goias State.

\section{Resumen}

El presente documento esboza las perspectivas y desafíos para la aplicación de un Pago por Servicios Ambientales (PSA) en el Estado de Goiás - Brasil, centrándose en la aplicación concreta de un mecanismo que está en un gran debate en este momento bajo la Convención sobre Cambio Climático: Reducción de emisiones por Deforestación y Degradación Forestal (Reducing Emissions from Deforestation and Forest Degradation - REDD, en inglés). En um enfoque cualitativo, se utiliza de diversas fuentes bibliográficas (secundaria), para discutir el tema tratado. Y llegamos a la conclusión de que, a pesar de los retos y dificultades técnicas y operativas que aún deben ser abordados y resueltos, REDD puede ser factible y eficaz como mecanismo de reducción compensada de la deforestación y la conservación del Cerrado em el Estado de Goiás.

Palabras clave: el cambio climático, REDD, el bioma del Cerrado, el estado de Goiás. 
Introdução

A crise do sistema econômico-financeiro iniciada no fim de 2008, e que ainda se alastra pelos quatro cantos do mundo, não deixa dúvidas de que "outro mundo é urgente", parafraseando o mote entoado no Fórum Social Mundial. Para Dupas (2008), além do caráter estritamente econômico e financeiro, a crise também evidencia a quebra de legitimidade e de sustentação política, social e ambiental do sistema de produção e consumo da moderna sociedade urbano-industrial.

Os avanços alcançados pelo desenvolvimento científico e tecnológico em todas as áreas do conhecimento humano sem dúvida colocaram a humanidade diante de uma infinidade de informações, técnicas, equipamentos e objetos até pouco tempo inimagináveis, em termos de bem-estar social. Por outro lado, é também indiscutível que todos esses avanços e melhorias, além de não serem compartilhados por todos, acabaram gerando degradação e poluição ambiental, que numa escala exponencialmente crescente coloca em risco os ecossistemas naturais e a própria continuidade da vida dos seres humanos no planeta Terra.

Neste contexto, enxergar as potencialidades de uma estratégia econômica que valorize os recursos ambientais existentes pode contribuir para a operacionalização do conceito de desenvolvimento sustentável. Para ajudar nesta reflexão e tendo como base uma revisão bibliográfica representativa, algumas questões são apresentadas neste texto: o contexto das discussões atuais sobre as mudanças climáticas; o destaque do Brasil nessa discussão; a crescente importância atribuída aos conceitos de economia verde e de pagamento por serviços ambientais, tendo como pano de fundo o mecanismo de valoração econômica do desmatamento evitado e o Cerrado como possível protagonista desta nova ótica.

\section{Mudanças climáticas e a importância das florestas: 0 contexto do REDD}

O tema das mudanças climáticas consegue expressar um dos muitos sintomas advindos do uso excessivo e inadequado dos recursos naturais de nossa sociedade, principalmente de combustíveis fósseis: carvão, petróleo e gás. Origina-se da evolução conjunta do crescimento econômico e das emissões de gases de efeito estufa (GEE) e se estende em problemas ambientais complexos e de difícil manejo, afetando também a economia 
e as sociedades do mundo todo. Podemos dizer que o problema do clima provavelmente é o desafio mais significativo do século XXI, pois é um tema que impacta diretamente a economia global, já que grande parte da solução passa pela alteração da matriz energética nos países, na busca de novas fontes de energia (renováveis) e de eficiência energética (Giddens, 2010; Abranches, 2010).

As alterações climáticas antropogênicas estão agora para além de qualquer dúvida, principalmente a partir da divulgação do quarto relatório de avaliação do Painel Intergovernamental sobre Mudança Climática $^{1}$ (Intergovernmental Panel on Climate Change - IPCC, em inglês), no qual aponta-se para a certeza científica - maior de 90\% -, quanto à responsabilidade das atividades humanas por essas alterações (IPCC, 2000). As evidências do efeito antrópico na mudança do clima são baseadas no aumento histórico das concentrações de GEE desde o início da Revolução Industrial, especialmente de dióxido de carbono $\left(\mathrm{CO}_{2}\right)$, metano $\left(\mathrm{CH}_{4}\right)$ e óxido nitroso $\left(\mathrm{N}_{2} \mathrm{O}\right)$. No caso do $\mathrm{CO}_{2}$, antes da Revolução Industrial sua concentração na atmosfera variou entre 170 e 280 partes por milhão (ppm) e hoje encontra-se em 387 ppm, um aumento, no mínimo, de 40\% (Raupach et al., 2007).

Com o aumento dessas partículas no ar, registros indicam um acréscimo de temperatura em média de $0,8^{\circ} \mathrm{C}$ desde os níveis pré-industriais, e estima-se que a temperatura média do planeta poderá elevar-se de 2,5 a $7^{\circ} \mathrm{C}$ nos próximos 100 anos (IPCC, 2007). Alguns impactos atuais ou do futuro próximo do aquecimento global já são considerados irreversíveis, pelo próprio caráter inercial do sistema climático, como nos lembra Matthews e Caldeira (2008), pois mesmo estabilizando hoje as emissões de GEE, elas permanecerão atuando por décadas, em decorrência das emissões que ocorreram no passado.

$\mathrm{O}$ aumento de $0.8^{\circ} \mathrm{C}$ na temperatura global pode parecer pouco, mas como o equilíbrio climático do planeta é frágil, estão sendo observados efeitos importantes causados por ele, tais como furacões, tornados, secas prolongadas, ondas de calor, chuvas intensas, entre muitos outros (IPCC, 2000). Para Myers (2002), a saúde pública poderá ser afetada com o aumento na frequência de doenças relacionadas ao calor e de áreas sujeitas à dengue e febre amarela, por exemplo. As mudanças no regime de chuvas podem ameaçar seriamente a segurança alimentar de grandes populações e a oferta de água, fazendo com que contingentes populacionais se desloquem, migrando para outras regiões. 
No âmbito da Convenção-Quadro das Nações Unidas sobre Mudança do Clima (CQNUMC), ${ }^{2}$ a operacionalização de mecanismos e processos que viabilizem e estimulem reduções de emissões de GEE estão sendo discutidas e adotadas por instituições, empresas e governos do mundo todo (Stern, 2007). A confecção de inventários de GEEs, a implementação de políticas públicas e regulamentações que limitem as emissões diretamente, ou que criem incentivos para melhor eficiência dos setores energético, industrial, de transporte e agropecuário, que promovam um maior uso de fontes renováveis de energia ou ainda que estimulem o estabelecimento de mecanismos de flexibilização estabelecidos pelo Protocolo de Quioto (comércio de emissões, implementação conjunta, mecanismo de desenvolvimento limpo) são exemplos dessas medidas.

Ademais, com a publicação do Relatório Stern ${ }^{3}$ em 2006 e do IV Relatório do IPCC em 2007 - onde foram apontadas que as emissões de carbono provenientes do desmatamento e da degradação florestal contribuíam com 17\% das emissões mundiais de GEEs e que o combate a essas emissões seria a forma mais fácil e barata de minimizar os efeitos das mudanças climáticas -, começou a ser pensado e discutido na Convenção do Clima outro mecanismo que permitisse englobar os países em desenvolvimento, detentores de florestas tropicais, na tarefa de redução das emissões de GEE. Assim, desde a realização em 2007 da $13^{\text {a }}$ Conferência das Partes (COP 13), em Bali, outro instrumento que vem ganhando destaque e apoio nas negociações capitaneadas pela CQNUMC é aquele conhecido como Redução de Emissões por Desmatamento e Degradação Florestal (Reducing Emissions from Deforestation and Forest Degradation - REDD, em inglês). A sigla foi modificando-se ao longo dos anos nas negociações, e quando o mecanismo foi proposto incluía apenas a redução do desmatamento (RED). Conforme a inclusão de outras atividades, como a questão da degradação florestal, a sigla foi ampliada para REDD. O REDD+ inclui o papel da conservação, o manejo florestal sustentável e o aumento dos estoques de carbono, e o REDD++ (embora ainda seja uma visão de longo prazo) inclui também o setor da agricultura, florestas e outros usos da terra.

Para Parker et al. (2008), a ideia é criar valores econômicos para a floresta em pé, ou para o desmatamento evitado, como tem sido chamado. É o papel da valoração dos serviços ambientais proporcionados pelas florestas como instrumento de política pública. Além de capturar e armazenar o carbono, as florestas são importantes para a manutenção do sistema 
climatológico e do ciclo hidrológico; conseguem conter as queimadas; ajudam na reciclagem de nutrientes, no controle de erosões e na proteção da biodiversidade, sendo também abrigo para a fauna; e, por fim, são importantes porque fornecem recursos que apoiam a nossa subsistência (madeira, alimentos, essências etc.) e o bem-estar físico e cultural (Angelsen, 2008). Desta forma, o REDD pode vir a ser uma alternativa rentável para reduzir o desmatamento, evitar queimadas e a degradação florestal, e promover a proteção florestal, ao mesmo tempo que pode oferecer novas fontes de renda às populações, principalmente aquelas que vivem nas zonas rurais e naturais.

Desde então, o conceito REDD vem ganhando muito apoio, e existe um consenso geral de que, embora não tenha um formato estabelecido ainda existem muitas discussões de como deve funcionar no que tange a processos e financiamento -, o mecanismo será incorporado em um futuro acordo climático. Diferentemente do Mecanismo de Desenvolvimento Limpo (MDL), que não inclui as florestas naturais remanescentes, o REDD propõe compensações financeiras aos agentes que se prontificarem a proteger suas florestas. Em 2010, na Conferência das Partes ocorrida em Cancun (COP 16), foram aprovados o conceito, as diretrizes, as salvaguardas e as principais regras para REDD, sendo que agora é um mecanismo de mitigação voluntário dos países em desenvolvimento no âmbito da CQNUMC e conta com o apoio técnico e financeiro dos países desenvolvidos. Esse é o modelo do Fundo Amazônia, ${ }^{4}$ constituído pelo governo brasileiro para internalizar divisas no contexto REDD.

Outra possibilidade de financiar REDD são os mecanismos de mercado. A exemplo de outros mercados, um poluidor poderá compensar suas emissões comprando créditos de quem ainda tem o que conservar (governo, proprietários de terras, povos tradicionais). Ou seja, os países com interesses globais de redução de emissão de carbono podem pagar governos nacionais para, por sua vez, persuadir um subconjunto de agentes locais a desmatar menos (Wunder, 2005). As duas opções de fontes de financiamento também são possíveis, e os países avançam na definição de marcos regulatórios que permitam a utilização do REDD.

Transição para uma economia verde: pagamentos por serviços ambientais (REDD)

O Brasil, além de abrigar a maior área de floresta tropical do planeta e ser o segundo país em extensão de florestas, também se posiciona 
entre os quatro maiores emissores globais de GEE, estando atrás apenas da China, dos Estados Unidos e da União Europeia. As emissões brasileiras se distinguem dos três maiores emissores em um aspecto crucial: por aqui a queima de combustíveis fósseis não é o maior vilão do efeito estufa, e sim o setor chamado de "mudança do uso da terra", principalmente pelo desmatamento e pelas queimadas de áreas para a conversão em pastagens e/ou lavouras. De acordo com o último Inventário Brasileiro de Emissões de GEE (MCT, 2010), que abrange o período de 1990 a 2005, esse setor respondeu por $75 \%$ das emissões nacionais, em particular da conversão de florestas para uso agropecuário, principalmente nos biomas da Amazônia e do Cerrado.

Para reduzir o seu papel no aquecimento do planeta, o Brasil apresentou em 2009, na $15^{\text {a }}$ Conferência das Partes (COP-15), as metas nacionais voluntárias de redução das emissões de GEEs, entre 36,1\% e 38,9\%, projetadas até 2020, também definidas na recentemente aprovada Política Nacional sobre Mudança do Clima (Brasil, 2009). Entre os instrumentos utilizados para alcançar essas metas estão os Planos de Ação para a Prevenção e Controle do Desmatamento e das Queimadas nos biomas da Amazônia (PPCDAm) - que prevê a redução de pelo menos $80 \%$ da taxa de desmatamento - e do Cerrado (PPCerrado), no qual consta, entre os objetivos, o de reduzir em 40\% o desmatamento na região até 2020.

Desta forma, para ajudar na meta nacional de redução das emissões de GEE e, ao mesmo tempo, progredir no estabelecimento de um desenvolvimento econômico de baixa emissão de carbono, é inegável a importância da inserção dos conceitos e preceitos da economia verde ${ }^{5}$ na discussão e proposição de políticas públicas brasileiras. O PNUMA (2011) define economia verde como uma economia que resulta em "melhoria do bem-estar da humanidade e igualdade social, ao mesmo tempo em que reduz significativamente riscos ambientais e escassez ecológica”. Isso nada mais significa do que ter uma economia em que o crescimento de renda e emprego seja direcionado, por investimentos públicos e privados, à promoção de atividades de baixa emissão de carbono (que reduzem as emissões) e ao aumento da eficiência energética e o uso sustentável de recursos ambientais.

No cenário de crise planetária, a compensação econômica para a manutenção dos serviços ambientais pode contribuir, definitivamente, para alcançar processos mais sustentáveis de desenvolvimento, em suas 
diversas dimensões: econômica, social e ambiental (TEEB, 2011). Com recursos naturais abundantes, apesar de ameaçados, o Brasil poderá se destacar pelo uso eficiente e pela conservação dos seus recursos naturais, gerando riquezas, renda, crescimento mais inclusivo e a preservação do bem comum ambiental. Já que o desmatamento figura entre os principais agentes catalisadores das emissões no nosso país, a remoção de incentivos perversos (créditos subsidiados, incentivos fiscais, construção de estradas etc) e a criação de políticas que valorizem nossos recursos ambientais, transformando definitivamente o capital natural em um ativo, podem contribuir para a transição rumo a uma economia verde. Nestes termos, o pagamento por serviços ambientais (PSA), principalmente aqueles advindos da gestão sustentável e conservação de nossas florestas (REDD), poderá assumir um papel importante nessa transição.

\section{REDD: princípios e desafios}

Apesar de um acordo com REDD ainda não ter sido assinado na Convenção do Clima, já existem projetos em curso em diversos países e várias propostas sobre o escopo das suas atividades ${ }^{6}$ vêm sendo submetidas e recomendadas ao órgão técnico da Convenção, denominado Órgão Subsidiário de Assessoramento Científico e Tecnológico (SBSTA). É consenso que algumas questões devem ser observadas dentro desses mercados/contratos. Para Wunder (2005), um conjunto diz respeito à adicionalidade, ao vazamento e à permanência. A adicionalidade pode ser definida como o grau de sucesso de um programa de PSA em aumentar a provisão de serviços ambientais, em comparação com um cenário sem PSA. Por isso, existe a necessidade de estabelecer uma linha de base ${ }^{7}$ (cenário de referência) da provisão de serviços, para daí determinar o quanto será provido adicionalmente devido ao pagamento. Outro aspecto é a questão do vazamento, um efeito de deslocamento espacial de alguma atividade, por exemplo, o desmatamento, por causa de uma intervenção de PSA. No caso, o desmatamento iria para áreas não cobertas pelo esquema de PSA. Por fim, a permanência se refere à análise da continuidade na provisão dos serviços ao longo do tempo e sua potencial reversibilidade.

Ademais, outros critérios técnicos devem nortear as discussões sobre REDD. Eles dizem respeito ao emprego de metodologias para monitoramento, relatório e verificação (MRV) dos sistemas de emissões (Grassi 
et al., 2008). Um sistema de monitoramento bem elaborado é peça-chave para contabilizar as reduções de emissão ou o aumento dos estoques de carbono (e as incertezas a eles associadas) em relação a um nível de referência pré-determinado. O relatório depende da solidez das metodologias utilizadas, da credibilidade das estimativas que delas resultam e da forma como a informação é compilada e apresentada. O propósito da verificação é avaliar se a informação está bem documentada, explicada e se se baseia em metodologias robustas (Grassi et al., 2008).

Por fim, aspectos socioambientais devem ser observados, assegurando a participação pública, os interesses e os direitos dos povos indígenas e comunidades tradicionais (Bonfante et al., 2010), pois o envolvimento de todas as partes interessadas, especialmente daqueles que vivem na floresta ou em seu entorno, é peça fundamental para que a sustentabilidade ambiental e social das políticas para reduzir o desmatamento tenha sucesso.

\section{Oportunidades e desafios do REDD no contexto do Cerrado}

O Cerrado, apesar de ser um grande emissor de carbono, com cerca de 302 milhões de toneladas de $\mathrm{CO}_{2}$ sendo emitidos em 2005 (MCT, 2010), também é um grande sumidouro. Diferentemente da Amazônia, a maior parte da biomassa do Cerrado está no subsolo, podendo constituir até $70 \%$ da biomassa total, dependendo da vegetação dominante (Castro; Kauffman, 1998). Desta forma, o estoque de carbono no bioma é de cerca $29 \mathrm{t} /$ ha na vegetação e $117 \mathrm{t} /$ ha no solo, até um metro de profundidade (IPCC, 2000). Outros autores corroboram essa visão (Bustamante; Ferreira, 2010; Bustamante et al., 2006; Abdala, 1993), mostrando a importância do solo como reservatório de carbono e confirmando o potencial de implementação de mecanismos do tipo REDD nessa região brasileira, principalmente se a abrangência do mecanismo aumentar, para incluir o setor agrícola (REDD++).

Contrariamente às práticas de manejo usuais da agricultura e pecuária intensivas, que promovem a perda excessiva do carbono orgânico do solo quando do seu preparo convencional, o uso de sistemas de manejo sustentáveis (plantio direto, integração lavoura-pecuária) e a recuperação de áreas degradadas têm sido apontados como mitigadores das emissões de GEE, além de evitar o processo de degradação ambiental e a demanda 
pela abertura de novos espaços para a produção no Cerrado. O Programa de Agricultura de Baixo Carbono ${ }^{8}$ (ABC), lançado pelo Ministério da Agricultura em 2010, vai nessa direção, integrando as iniciativas setoriais para que o país atinja as metas voluntárias de redução das emissões de GEE.

No bioma Cerrado, em apenas 40 anos, dos seus 2,06 milhões de quilômetros quadrados originais, menos da metade ainda está de pé, convertidos em áreas para pastagens cultivadas e culturas agrícolas (Sano et al., 2007). Observa-se o avanço recente da fronteira agropecuária nos estados ao norte do bioma, como Tocantins, Maranhão, Piauí e Bahia, justamente em áreas de remanescentes. E estudos indicam que aproximadamente 40 milhões de hectares do Cerrado ainda possuem áreas "favoráveis" para a agricultura, constituídos por terrenos com declividade favorável à mesma (80\% entre 0 e $5^{\circ}$ ) (Ferreira et al., 2012). Por outro lado, ainda não conseguimos alcançar o mínimo de $10 \%$ de áreas protegidas estipulado pela Convenção sobre Diversidade Biológica (CDB), índice atrelado à sobrevivência de inúmeras espécies de animais e plantas e à manutenção de serviços ambientais (Klink; Machado, 2005).

Para o Ministério do Meio Ambiente (MMA, 2011), caso os níveis de desmatamento no Cerrado forem mantidos, o bioma passará a assumir papel de destaque nas emissões nacionais de GEE, dado o seu elevado potencial de emissões, tanto na vegetação como no solo. E mesmo com o compromisso assumido em Copenhague, de redução de 40\% do desmatamento até 2020, $8.000 \mathrm{~km}^{2}$ de Cerrado ainda estariam disponíveis para a conversão. Ou seja, o Cerrado tem todo um cenário potencial para a implementação de projetos de REDD.

Por outro lado, o estado de Goiás, por abrigar o bioma Cerrado em 98\% do seu território, é representante fiel deste contexto de intensa conversão e pouca proteção efetiva das áreas de vegetação remanescente. Cerca de 63\% de sua vegetação natural já foi desmatada para a implantação de pastagens e agricultura (Sano et al., 2006) e as áreas destinadas à proteção integral (parques) não somam 1\% do seu território. Tanto por sua importância econômica (celeiro do agronegócio) e ambiental (berço de biodiversidade e águas), Goiás pode ter um papel central a desempenhar no cenário das mudanças climáticas. Partindo-se do princípio de que a maior parte da contribuição das emissões de GEEs no estado esteja no setor agropecuário, pensar em políticas públicas eficazes, do ponto de vista ambiental e ao mesmo tempo econômica e politicamente atraentes para esses setores visando suas reduções, é peça fundamental. 
Os desafios são imensos, mas as possibilidades são reais. Apesar de ainda não existir um acordo global para REDD, nem as bases legais no Brasil, certamente em um novo acordo climático as florestas entrarão como complementaridade aos mecanismos já existentes de reduções de GEE. Acordos bilaterais, nacionais e subnacionais também podem ser criados enquanto avançam as negociações na CQNUMC. Mas a regulamentação do sistema é imprescindível para dar segurança jurídica e administrativa, permitindo os efeitos desejados de REDD.

Atualmente encontra-se em tramitação no Congresso Nacional um projeto de lei federal (PL 5586/2009) que prevê a criação de um sistema nacional de REDD+. Também já existe uma iniciativa nacional (Fundo Amazônia) e outras iniciativas estaduais para a construção de estratégias e programas relacionados ao tema (Amazonas, Mato Grosso, Pará, Acre), que podem ajudar no desenho das bases de REDD para o estado de Goiás. Iniciativas-piloto de projetos REDD, de diversos atores do setor privado e governo, já são realidade no Brasil. Hoje, segundo o MMA (2010) somam-se 21 iniciativas.

A articulação de diferentes fontes de recursos, sejam públicos ou privados, nacionais ou internacionais, parece ser a melhor solução para a diversificação dos financiamentos, tonando-os mais flexíveis. E o desenvolvimento de um arranjo institucional para coordenar as diferentes ações de REDD, inclusive dos fluxos financeiros, garantiria o seu respaldo público, além de estabelecer padrões e certificações cuidadosamente desenhados. Assim, o apoio e a articulação de alguns atores são fundamentais para a validação do REDD no estado de Goiás: agência de fomento e desenvolvimento; poder público estadual, como as secretarias de Gestão e Planejamento, da Agricultura e do Meio Ambiente e Recursos Hídricos; universidades e entidades representativas (associações de produtores rurais, comunidades de unidades de conservação).

O aparato instrumental necessário para o bom funcionamento de mecanismos de REDD, mostrando a capacidade de monitoramento e verificação do desmatamento, pode iniciar-se a partir do estabelecimento de uma base de dados com o compartilhamento de informações, envolvendo diferentes setores do governo, nas esferas federal e estadual. Sistemas de monitoramento do desmatamento desenvolvidos para o Cerrado, transparentes e tecnicamente consistentes, já existem. Ademais, pesquisadores da UnB, UFG e INPE já iniciaram discussões para o desenvolvimento de 
uma metodologia específica para o monitoramento da cobertura do bioma associada às variações de biomassa, como subsídio para a quantificação das emissões de $\mathrm{CO}_{2}$ oriundas do desmatamento (Ferreira; Bustamante, 2011). Mas ainda carecemos de dados sobre a trajetória de áreas desmatadas e preservadas no bioma, sobre os efeitos da sazonalidade climática - em que o papel do fogo, na época da seca, é crucial para a fixação e liberação de carbono - e sobre as variações de biomassa aérea e subterrânea nas diferentes formações vegetacionais, fundamentais para o estabelecimento de métodos padronizados para o desenvolvimento de linhas de base e sistemas mais eficazes de monitoramento.

A Secretaria de Estado do Meio Ambiente e dos Recursos Hídricos (Semarh, 2012) deu início a um processo licitatório de contratação de consultoria e assessoria jurídica e financeira para a elaboração e implementação de um marco regulatório, com seus respectivos arranjos institucionais, que amparem a formação de um mercado de transação de ativos ambientais, com foco em florestas (Reserva Legal, Unidades de Conservação/Biodiversidade e Carbono Florestal) e recursos hídricos, tendo em vista um modelo de desenvolvimento econômico de baixo carbono no estado de Goiás. Um passo importante para uma estratégia estadual de REDD.

\section{Considerações finais}

O mecanismo REDD apresenta-se como estratégia política de maior alcance para fornecer um valor econômico à conservação do bioma Cerrado. Tal mecanismo, se bem aproveitado, poderá reduzir a pressão da expansão agropecuária sobre novas áreas de Cerrado, representando a base de uma nova economia rural e regional de baixa emissão de carbono e de valorização de suas florestas e de toda a biodiversidade que nela estão depositadas. Ao mesmo tempo, pode contribuir com as metas nacionais de redução de GEE da PNMC e gerar divisas para o estado de Goiás.

As iniciativas em curso (universidades, governo) são essenciais para que Goiás prepare as suas bases para a consolidação de uma estratégia de REDD. Porém, desafios institucionais e dificuldades técnicas/metodológicas e operacionais ainda precisam ser enfrentados e superados. Certamente, outros estudos que abordem o desempenho deste instrumento político ambiental na conservação do bioma precisam ser elaborados. 
Por fim, o REDD só terá sucesso se for incluído em um pacote maior de políticas públicas que consigam influenciar os rumos dos modelos de desenvolvimento econômico adotados para a região, optando pela combinação de políticas de comando e controle, de desincentivos econômicos para atividades que levem ao desmatamento e de outros incentivos positivos de valoração dos recursos naturais.

\section{Notas}

1. O IPCC foi estabelecido pela Organização Metereológica Mundial e pelo Programa das Nações Unidas para Meio Ambiente (UNEP) em 1988, para resumir o estado do conhecimento científico sobre mudança climática.

2. A Convenção Quadro das Nações Unidas sobre Mudança do Clima (CQNUMC), adotada em 1992 e ratificada em 1994 por mais de 175 países, objetiva estabilizar as emissões de gases de efeito estufa (GEE) pelos países, prevenindo uma interferência humana perigosa para o clima do planeta. O Protocolo de Quioto regulamenta os compromissos legalmente assumidos na Convenção e foi assinado em 1997, entrando em vigor em 2005. Nele são estabelecidos compromissos legalmente vinculativos de redução de emissões de GEE para 37 países desenvolvidos, com exceção dos Estados Unidos.

3. Sir Nicholas Stern analisa os efeitos na economia mundial das alterações climáticas e chega à conclusão de que, com um investimento de apenas $1 \%$ do PIB Mundial, pode evitar-se a perda de $20 \%$ do mesmo PIB num prazo de simulação de 50 anos.

4. Disponivel em: http://www.fundoamazonia.gov.br/FundoAmazonia/fam/ site_pt/Esquerdo/Fundo/.

5. Um dos temas centrais da Rio+20, a Conferência das Nações Unidas sobre Desenvolvimento Sustentável.

6. Uma síntese dessas discussões está sumariada em $O$ Pequeno Livro Vermelho do REDD. Disponível em: http://www.ipam.org.br/biblioteca/livro/ O-Pequeno-Livro-do-REDD-/336.

7. Já existem metodologias, apesar de controversas, para o estabelecimento de linhas de base, principalmente para projetos relacionados à captura de carbono. As mais usadas contemplam as médias de desmatamento passado de cinco a dez anos. Porém, uma metodologia mais consistente, acordada internacionalmente, está sendo buscada.

8. Disponível em: http://www.agricultura.gov.br/abc/.

\section{Referências}

ABDALA, G. C. Análise energética de um cerrado e sua exploração por atividade de carvoejamento rústico. Dissertação (Mestrado em Ecologia) - Instituto de Ciências Biológicas, Universidade de Brasília, Brasília, 1993. 
ABRANCHES, S. Copenhague: antes e depois. Rio de Janeiro: Civilização Brasileira, 2010.

ANGELSEN, A. (Org.). Moving Ahead with REDD: Issues, Options and Implications. Poznan, Polônia: CIFOR, 2008.

BRASIL. Lei n. 12.187, de 29 de dezembro de 2009. Institui a Política Nacional sobre Mudança do Clima - PNMC e dá outras providências.

BONFANTE, T. M.; VOIVODIC, M.; FILHO, L. M. Desenvolvendo Salvaguardas Socioambientais de REDD+: um guia para processos de construção coletiva. Piracicaba, SP: Imaflora, 2010.

BUSTAMANTE, M. M. C. et al. Soil carbon storage and sequestration potencial in the cerrado region of Brazil. In: Carbon Sequestration in Soils of Latin America. The Haworth Press, p. 285-304, 2006.

BUSTAMANTE, M. M. C.; FERREIRA JUNIOR, L. G. Land use change and the carbon budget in the Brazilian Cerrado (IN PRESS). In: HILL, Michael J.; HANAN, Niall P. (Org.). Ecosystem Function in Savannas: Measurement and Modeling at Landscape to Global Scales. Taylor \& Francis Group, 2010. v. 1, p. 111-111.

CASTRO, E. A.; KAUFFMAN, J. B. Ecosystem structure in the Brazilian Cerrado: a vegetation gradient of aboveground biomas, root mass and consumption by fire. Journal of Tropical Ecology, v. 14, p. 263-283, 1998.

DUPAS, G. O impasse ambiental e a lógica do capital. In: DUPAS, Gilberto (Org.). Meio ambiente e crescimento econômico: tensões estruturais. São Paulo: Ed. Unesp, 2008.

FERREIRA, L. G.; BUSTAMANTE, M. M. C. (Org.). Monitoramento das Emissões de Carbono no Cerrado Brasileiro. Embaixada Britânica, Brasília, 2011.

GIDDENS, A. A política da mudança climática. Rio de Janeiro: Jorge Zahar, 2010.

GRASSI, G. et al. Applying the conservativeness principle to REDD to deal with the uncertainties of the estimates. Environmental Reserch Lettes, 2008. 3 DOI:10.1088/1748-9326/3/3/035005.

IPCC - Intergovernmental Panel on Climate Change. The Physical Science Basis. Contribution of Working Group I to the Fourth Assessment Report of the IPCC. Cambridge, UK: Cambridge University Press, 2007.

IPCC - Intergovernmental Panel on Climate Change. Land use, land use change and forestry. Cambridge, UK: Cambridge University Press, 2000

KLINK, C A; MACHADO, R. B. Conservation of the Brazilian Cerrado. Conservation Biology, v. 19, n. 3, p. 707-713, 2005.

FERREIRA, M. E. et al. Modeling Landscape Dynamics in the Central Brazilian Savanna Biome: future scenarios and perspectives for conservation, Journal of Land Use Science. Available online: 15 mar. 2012.

MATTHEWS, H. D.; CALDEIRA, K. Satabilizing Climate Requires Near-Zero Emissions. Geophysical research Letters, v. 35, p. L04705-doi: 10.1029/2007GL032388, 2008.

MCT - Ministério da Ciência e Tecnologia. Segundo Inventário Brasileiro de Emissões e Remoções Antrópicas de Gases de Efeito Estufa. Relatório de Referência. 2010. 
MYERS, N. Environmental Refugees: a Growing Phenomenon of the $21^{\text {st }}$ Century. Philosophical Transactions of the Royal Society, v. B357, n. 1420, p. 609-13, 2002.

MMA - Ministério do Meio Ambiente. Experiências Brasileiras em REDD. Serviço Florestal Brasileiro, 2010.

MMA - Ministério do Meio Ambiente. Plano de Ação para Prevenção e Controle do Desmatamento e das Queimadas no Cerrado: Conservação e Desenvolvimento. Brasília, 2011.

PARKER, C. et al. The Little REDD Book: A guide to governmental and nongovernmental proposals for reducing emissions from deforestation and degradation. Oxford, UK: The Global Canopy Foundation, 2008.

PNUMA - Programa das Nações Unidas para o Meio Ambiente. Caminhos para o Desenvolvimento Sustentável e a Erradicação da Pobreza: síntese para tomadores de decisão, 2011.

RAUPACH, M. R. et al. Global and Regional Drivers of a Accelerating $\mathrm{CO}_{2}$ emissions. Proceedings of the National Academy of Sciences, v. 104, n. 24, 2007.

SANO, E. E. et al. Padrões de cobertura de solos do Estado de Goiás. In: FERREIRA, L. G. (Org.). Conservação da Biodiversidade e Uso Sustentável em Goiás. Estratégias, Prioridades e Perspectivas. Goiânia: SEMARH/Agência Ambiental/ Banco Mundial. 2006.

SANO, E. E. et al. Mapeamento de cobertura vegetal do bioma Cerrado: estratégias e resultados. Planaltina - DF: Embrapa Cerrados ISSN 1517-5111. n. 190, 2007.

SEMARH - Secretaria de Estado do Meio Ambiente e dos Recursos Hídricos. Núcleo de Projetos Especiais. Projeto Cerrado Sustentável Goiás. Termo de Referência n. 002/2010 - UCP/NPE/SEMARH.

STERN, N. The Economics of Climate Change: The Stern Review. Cambridge, UK: Cambridge University Press, 2007.

TEEB - The economics of ecosystems an biodiversity: mainstreaming the economics of nature: a synthesis of the approach, conclusions and recommendations of TEEB. 2011.

WUNDER, S. Payments for environmental services: some nuts and bolts. In: CIFOR Occasional Paper. CIFOR, v. 42, 2005.

Juliana Ferreira Leite - Doutoranda em Geografia pela Universidade Federal de Goiás.

Laerte Guimarães Ferreira - Doutor pela University of Arizona - Professor da Universidade Federal de Goiás.

Recebido para publicação em junho de 2012

Aceito para publicação em setembro de 2012 